\title{
Magnetohydrodynamic waves and their stability status in solar spicules
}

\author{
I. Zhelyazkov
}

Faculty of Physics, Sofia University, 5 James Bourchier Blvd., 1164 Sofia, Bulgaria

e-mail: izh@phys.uni-sofia.bg

Received 28 July 2011 / Accepted 14 October 2011

\begin{abstract}
Aims. We investigate conditions under which magnetohydrodynamic waves propagating along spicules become unstable because of the Kelvin-Helmholtz instability.

Methods. We employ the dispersion relations of normal modes (kink and sausage waves) derived from the linearised magnetohydrodynamic equations. We assume real wave numbers and complex angular wave frequencies, namely complex wave phase velocities. The dispersion relations are solved numerically at fixed input parameters and various flow velocities.

Results. It is shown that the stability of the waves depends upon three parameters, the density contrast between spicules and their environment, the ratio of the background magnetic field outside to that inside spicules, and the value of the Alfvén-Mach number (the ratio of the jet velocity to Alfvén speed inside the spicules). At certain densities and magnetic fields, an instability of the Kelvin-Helmholtz type can arise if the Alfvén-Mach number exceeds a critical value - in our case it is equal to 12.6, which means that for an Alfvén speed inside the spicules of $70 \mathrm{~km} \mathrm{~s}^{-1}$ the jet velocity should be larger than $882 \mathrm{~km} \mathrm{~s}^{-1}$.

Conclusions. It is found that only kink waves can become unstable, while the sausage ones are always unaffected by the Kelvin-Helmholtz instability.
\end{abstract}

Key words. methods: numerical - Sun: filaments, prominences - magnetohydrodynamics (MHD) - Sun: chromosphere - waves instabilities

\section{Introduction}

One of the most enduring mysteries in solar physics is why the Sun's outer atmosphere, or corona, is millions of kelvins hotter than its surface. Among the proposed theories for coronal heating is one that considers the role of spicules - narrow jets of plasma shooting up from just above the Sun's surface - in that process (see Athay \& Holzer 1982 and Athay 2000). For decades, it had been assumed that spicules might be sending heat into the corona. However, observational research in the 1980 s found that spicule plasma did not reach coronal temperatures, and this line of study largely fell out of vogue. Kukhianidze et al. (2006) were the first to report the observation of kink waves in solar spicules - their wavelength was found to be $\sim 3500 \mathrm{~km}$, and the period of waves was estimated to be in the range of 35-70 s. These authors argued that these waves may carry photospheric energy into the corona and therefore might be of importance to coronal heating. Zaqarashvili et al. (2007) analysed consecutive height series of $\mathrm{H} \alpha$ spectra in solar limb spicules at the heights of $3800-8700 \mathrm{~km}$ above the photosphere, and detected Doppler-shift oscillations with periods of 20-25 and 75-110 s. According to authors, the oscillations could be caused by waves' propagation in thin magnetic flux tubes anchored in the photosphere. Moreover, observed waves can be used to improve our understanding of spicule seismology, and the magnetic field induction in spicules at the height of $\sim 6000 \mathrm{~km}$ above the photosphere is estimated to be 12-15 G. De Pontieu et al. (2007) identified a new class of spicules that moved much faster and were shorter lived than the traditional spicules, which have speeds of between 20 and $40 \mathrm{~km} \mathrm{~s}^{-1}$ and lifespans of from 3 to $7 \mathrm{~min}$.
These type II spicules, observed in Ca II $854.2 \mathrm{~nm}$ and $\mathrm{H} \alpha$ lines (see Sterling et al. 2010), are much more dynamic: they form rapidly (in $\sim 10 \mathrm{~s}$ ), are very thin $(\leqslant 200 \mathrm{~km}$ wide), have lifetimes of 10-150 s (at any one height), and shoot upwards at high speeds, often in excess of $100-150 \mathrm{~km} \mathrm{~s}^{-1}$, before disappearing. The rapid disappearance of these jets had suggested that the plasma they carried might get very hot, but direct observational evidence of this process had not been reported. Both types of spicules are observed to carry Alfvén waves with significant amplitudes of order $20 \mathrm{~km} \mathrm{~s}^{-1}$. De Pontieu et al. (2011) used new observations from the Atmospheric Imaging Assembly on NASA's recently launched Solar Dynamics Observatory and its Focal Plane Package for the Solar Optical Telescope (SOT) on the Japanese Hinode satellite. Their observations reveal "a ubiquitous coronal mass supply in which chromospheric plasma in fountainlike jets or spicules is accelerated upward into the corona, with much of the plasma heated to temperatures between $\sim 0.02$ and 0.1 million kelvin (MK) and a small but sufficient fraction to temperatures above $1 \mathrm{MK}$. These observations provide constraints on the coronal heating mechanism(s) and highlight the importance of the interface region between photosphere and corona". Nevertheless, Moore et al. (2011) from Hinode observations of solar X-ray jets, type II spicules, and granule-size emerging bipolar magnetic fields in quiet regions and coronal holes, advocate a scenario for powering coronal heating and the solar wind. In this scenario, type II spicules and Alfvén waves are generated by the granule-size emerging bipoles in the manner of the generation of X-ray jets by larger magnetic bipoles. From observations and this scenario, the authors estimate that type II spicules and their co-generated Alfvén waves carry into 
the corona an area-average flux of mechanical energy of $\sim 7 \times$ $10^{5} \mathrm{erg} \mathrm{s}^{-1} \mathrm{~cm}^{-2}$. This is enough to power the corona and solar wind in quiet regions and coronal holes, hence indicates that the granule-size emerging bipoles are the main engines that generate and sustain the entire heliosphere. The upward propagation of high- and low-frequency Alvfén waves along spicules detected from SOT's observations on Hinode was also reported by $\mathrm{He}$ et al. (2009) and Tavabi et al. (2011). He et al. (2009) found in four cases that the spicules are modulated by highfrequency $(\geqslant 0.02 \mathrm{~Hz})$ transverse fluctuations. These fluctuations are suggested to be Alfvén waves that propagate upwards along the spicules with phase speeds ranging from 50 to $150 \mathrm{~km} \mathrm{~s}^{-1}$. Three of the modulated spicules display clear wave-like shapes with short wavelengths shorter than $8 \mathrm{Mm}$. We note also that Kudoh \& Shibata (1999) presented a torsional Alfvén-wave model of spicules (actually the classical type I spicules) and discussed the possibility of wave coronal heating - they estimated that the energy flux transported into corona is of about $3 \times 10^{5} \mathrm{erg} \mathrm{s}^{-1} \mathrm{~cm}^{-2}$, i.e., roughly half of the flux carried by the Alfvén waves running on type II spicules (see Moore et al. 2011). Tavabi et al. (2011) performed a statistical analysis of the SOT/Hinode observations of solar spicules and their wavelike behaviour, and argued that there is a possible upward propagation of Alfvén waves inside a doublet spicule with a typical wave's period of $110 \mathrm{~s}$.

Since spicules support Alfvén (or more generally magnetohydrodynamic) wave propagation, it is of great importance to determine their dispersion characteristics and more specifically their stability/instability status. If while propagating along the jets, MHD waves become unstable and the expected instability is of the Kelvin-Helmholtz type, that instability can trigger the onset of wave turbulence leading to an effective plasma jet heating and the acceleration of the charged particles. We note that the Alfvénic turbulence is considered to be the most promising source of heating in the chromosphere and extended corona (van Ballegooijen et al. 2011). In this study, we investigate these travelling wave properties for a realistic, cylindrical geometry of the spicules considering appropriate values for the basic plasma jet parameters (mass density, magnetic fields, sound, Alfvén, and jet speeds), as well as those of the environment. For detailed reviews of the oscillations and waves in magnetically structured solar spicules, we refer the reader to Zaqarashvili \& Erdélyi (2009) and Zaqarashvili (2011). Our research concerns the dispersion curves of kink and sausage modes for the MHD waves travelling primarily along the type II spicules for various values of the jet speed. In studying wave propagation characteristics, we assume that the axial wave number $k_{z}$ ( $\hat{z}$ is the direction of the embedded constant magnetic fields in the two media) is real, while the angular wave frequency, $\omega$, is complex. The imaginary part of that complex frequency is the wave growth rate when a given mode becomes unstable. All of our analysis is based on a linearised set of equations for the adopted form of magnetohydrodynamics. We show that the stability/instability status of the travelling waves depends entirely on the magnitudes of the flow velocities and the values of two important control parameters, namely the so-called density contrast (the ratio of the mass density inside to that outside the flux tube) and the ratio of the background magnetic field of the environment to that of the spicules themselves.

In the next section, we specify the geometry and basic parameters of type II spicules, as well as the governing MHD wave equations and corresponding dispersion relations. Section 3 deals with their numerical solutions and discusses the derived dispersion curves and growth rates when the waves are unstable. The last Sect. 4 summarises the main results derived in this paper.

\section{Geometry, the basic MHD equations, and the wave dispersion relations}

The simplest model of spicules is a straight vertical cylinder with radius $a$ filled with ideal compressible plasma of density $\rho_{\mathrm{i}} \sim 3 \times$ $10^{-13} \mathrm{~g} \mathrm{~cm}^{-3}$ (Sterling 2000) and immersed in a constant magnetic field $\boldsymbol{B}_{\text {i }}$ directed along the $z$ axis. Our flux tube can be considered as that in Fig. 3 of Matsumoto \& Shibata paper (2010) starting at the height of $2 \mathrm{Mm}$ from the tube footpoint. The mass density of the environment, $\rho_{\mathrm{e}}$, is much, say 50-100 times, less than that of the spicule, while the magnetic field induction $B_{\mathrm{e}}$ might be of the order of or less than $B_{\mathrm{i}} \sim 10-15 \mathrm{G}$. We note that while the parameters of classical type I spicules are welldocumented (Beckers 1968, 1972), those of type II spicules are generally disputed - Centeno et al. (2010), for example, on using a novel inversion code for Stokes profiles caused by the joint action of atomic level polarization and the Hanle and Zeeman effects to interpret the observations, claim that magnetic fields as strong as $\sim 50 \mathrm{G}$ were detected in a very localized area of the slit, which might represent a lower field strength for the organized network spicules.

Assuming that the density contrast - the ratio of equilibrium plasma density outside to that inside of spicule - is $\eta=0.01$, our choice of the sound and Alfvén speeds in the jet is $c_{\mathrm{si}}=$ $10 \mathrm{~km} \mathrm{~s}^{-1}$ and $v_{\mathrm{Ai}}=70 \mathrm{~km} \mathrm{~s}^{-1}$, respectively, while those speeds in the environment are correspondingly $c_{\mathrm{se}} \cong 605 \mathrm{~km} \mathrm{~s}^{-1}$ and $v_{\mathrm{Ae}}=250 \mathrm{~km} \mathrm{~s}^{-1}$. All these values are in agreement with the condition for the balance of total pressures at the flux tube interface - that condition can be expressed in the form

$p_{\mathrm{i}}+\frac{B_{\mathrm{i}}^{2}}{2 \mu}=p_{\mathrm{e}}+\frac{B_{\mathrm{e}}^{2}}{2 \mu}$,

which yields (Edwin \& Roberts 1983)

$\frac{\rho_{\mathrm{e}}}{\rho_{\mathrm{i}}}=\frac{c_{\mathrm{si}}^{2}+\frac{\gamma}{2} v_{\mathrm{Ai}}^{2}}{c_{\mathrm{se}}^{2}+\frac{\gamma}{2} v_{\mathrm{Ae}}^{2}}$.

Here $c_{\mathrm{s}}=\left(\gamma p_{0} / \rho_{0}\right)^{1 / 2}$ is the sound speed, where $\gamma=5 / 3$ is the polytropic index, $v_{\mathrm{A}}=B_{0} /\left(\mu \rho_{0}\right)^{1 / 2}$ is the Alfvén speed, and $\mu$ is the plasma permittivity. For simplicity in notation, $p_{0}, \rho_{0}$, and $B_{0}$ denote the equilibrium pressure, mass density, and magnetic field in either medium.

We assume that the magnetohydrodynamic, say Alfvén, waves are generated by random, turbulent-like, motions in the photosphere and that they propagate upwards in the flux tubes modelling the spicules. The basic equations governing the smallamplitude wave propagation are the linearised MHD equations for the perturbations of the fluid velocity $\boldsymbol{v}$, density $\rho$, pressure $p$, and magnetic field $\boldsymbol{B}$ defined in both media as

$\delta \boldsymbol{v}=\boldsymbol{v}-\boldsymbol{U}, \quad \delta \rho=\rho-\rho_{0}, \quad \delta p=p-p_{0}, \quad$ and $\delta \boldsymbol{B}=\boldsymbol{B}-\boldsymbol{B}_{0}$,

where $\boldsymbol{U}$ is the jet velocity, and $\rho_{0}, p_{0}$, and $\boldsymbol{B}_{0}$ as we have already mentioned are the equilibrium density, pressure, and background magnetic field, respectively. For convenience, we chose the frame of reference to be attached to the ambient medium. In this case

$\boldsymbol{U}^{\mathrm{rel}}=\boldsymbol{U}_{\mathrm{i}}-\boldsymbol{U}_{\mathrm{e}}$

is the relative flow velocity whose magnitude is a non-zero number inside the jet, and zero in the surrounding medium. For 
spicules, $U_{\mathrm{e}} \approx 0$, which is why the relative flow velocity is indeed the jet velocity, which we later denote as simply $\boldsymbol{U}$. Both the stream velocity $\boldsymbol{U}$ and magnetic field $\boldsymbol{B}_{0}$ are directed along the $z$ axis of the reference frame. Hence, the governing equations of the perturbations are

$$
\frac{\partial}{\partial t} \delta \rho+(\boldsymbol{U} \cdot \nabla) \delta \rho+\rho_{0} \nabla \delta \boldsymbol{v}=0
$$

$$
\begin{aligned}
\rho_{0} \frac{\partial}{\partial t} \delta \boldsymbol{v}+\rho_{0}(\boldsymbol{U} \cdot \nabla) \delta \boldsymbol{v} & +\nabla\left(\delta p+\frac{1}{\mu} \boldsymbol{B}_{0} \cdot \delta \boldsymbol{B}\right) \\
& -\frac{1}{\mu}\left(\boldsymbol{B}_{0} \cdot \nabla\right) \delta \boldsymbol{B}=0,
\end{aligned}
$$

$\frac{\partial}{\partial t} \delta \boldsymbol{B}+(\boldsymbol{U} \cdot \nabla) \delta \boldsymbol{B}-\left(\boldsymbol{B}_{0} \cdot \nabla\right) \delta \boldsymbol{v}+\boldsymbol{B}_{0} \nabla \cdot \delta \boldsymbol{v}=0$

$\frac{\partial}{\partial t} \delta p+(\boldsymbol{U} \cdot \nabla) \delta p+\gamma p_{0} \nabla \cdot \delta \boldsymbol{v}=0$

\section{$\nabla \cdot \delta \boldsymbol{B}=0$}

We note that the gravity force term in the momentum Eq. (2) has been omitted because one assumes that the mass density of the jet does not change appreciably in the limits of the spicule's length of the order of 10-11 Mm.

Assuming that all perturbations are proportional to $g(r) \exp \left[\mathrm{i}\left(-\omega t+m \phi+k_{z} z\right)\right]$ with $g(r)$ being just a function of $r$, and that in cylindrical coordinates the nabla operator has the form

$\nabla \equiv \frac{\partial}{\partial r} \hat{r}+\frac{1}{r} \frac{\partial}{\partial \phi} \hat{\phi}+\frac{\partial}{\partial z} \hat{z}$

from the above set of equations one can obtain a second order differential equation for the total pressure perturbation $\delta p_{\text {tot }}=$ $\delta p+\left(\boldsymbol{B}_{0} \cdot \boldsymbol{B}\right) / \mu$

$\left[\frac{\mathrm{d}^{2}}{\mathrm{~d} r^{2}}+\frac{1}{r} \frac{\mathrm{d}}{\mathrm{d} r}-\left(\kappa^{2}+\frac{m^{2}}{r^{2}}\right)\right] \delta p_{\mathrm{tot}}=0$,

as well as an expression for the radial component of the fluid velocity perturbation in terms of the first derivative of $\delta p_{\text {tot }}$

$\delta v_{\mathrm{r}}=-\frac{\mathrm{i}}{\rho_{0}} \frac{\omega-\boldsymbol{k} \cdot \boldsymbol{U}}{(\omega-\boldsymbol{k} \cdot \boldsymbol{U})^{2}-k_{z}^{2} v_{\mathrm{A}}^{2}} \frac{\mathrm{d}}{\mathrm{d} r} \delta p_{\text {tot }}$.

In Eq. (6), $\kappa$ is the so-called wave attenuation coefficient, which characterises the space structure of the wave and whose squared magnitude is given by the expression

$\kappa^{2}=-\frac{\left[(\omega-\boldsymbol{k} \cdot \boldsymbol{U})^{2}-k_{z}^{2} c_{\mathrm{s}}^{2}\right]\left[(\omega-\boldsymbol{k} \cdot \boldsymbol{U})^{2}-k_{z}^{2} v_{\mathrm{A}}^{2}\right]}{\left(c_{\mathrm{s}}^{2}+v_{\mathrm{A}}^{2}\right)\left[(\omega-\boldsymbol{k} \cdot \boldsymbol{U})^{2}-k_{z}^{2} c_{\mathrm{T}}^{2}\right]}$,

where

$c_{\mathrm{T}}=c_{\mathrm{s}} v_{\mathrm{A}} /\left(c_{\mathrm{s}}^{2}+v_{\mathrm{A}}^{2}\right)^{1 / 2}$

is the so-called tube velocity (Edwin \& Roberts 1983). As seen from the expressions for the attenuation coefficient and tube velocity, both quantities have different values inside and outside the flux tube owing to the different magnitudes of sound and Alfvén speeds in both media.
The differential Eq. (6) have two different solutions inside and outside the jet, notably

$\delta p_{\text {tot }}(r)=\left\{\begin{array}{c}A I_{m}\left(\kappa_{\mathrm{i}} r\right) \text { for } r \leqslant a, \\ B K_{m}\left(\kappa_{\mathrm{e}} r\right) \text { for } r \geqslant a,\end{array}\right.$

and, accordingly, different expressions for $\delta v_{\mathrm{r}}$

$\delta v_{\mathrm{r}}(r \leqslant a)=-\frac{\mathrm{i}}{\rho_{\mathrm{i}}} \frac{\omega-\boldsymbol{k} \cdot \boldsymbol{U}}{(\omega-\boldsymbol{k} \cdot \boldsymbol{U})^{2}-k_{z}^{2} v_{\mathrm{Ai}}^{2}} \kappa_{\mathrm{i}} A I_{m}^{\prime}\left(\kappa_{\mathrm{i}} r\right)$

and

$\delta v_{\mathrm{r}}(r \geqslant a)=-\frac{\mathrm{i}}{\rho_{\mathrm{e}}} \frac{\omega}{\omega^{2}-k_{z}^{2} v_{\mathrm{Ae}}^{2}} \kappa_{\mathrm{e}} B K_{m}^{\prime}\left(\kappa_{\mathrm{e}} r\right)$.

Here $I_{m}$ and $K_{m}$ are the modified Bessel functions of the first and second kind. After applying the boundary conditions to ensure the continuity of the total pressure perturbation $\delta p_{\text {tot }}$ and perturbed interface $\frac{\delta v_{\mathrm{r}}}{\omega-\boldsymbol{k} \cdot \boldsymbol{U}}$ (Chandrasekhar 1961), we arrive at the dispersion equations of kink $(m=1)$ and sausage $(m=0)$ surface modes (Terra-Homen et al. 2003; Nakariakov 2007)

$$
\begin{aligned}
\frac{\rho_{\mathrm{e}}}{\rho_{\mathrm{i}}}\left(\omega^{2}-k_{z}^{2} v_{\mathrm{Ae}}^{2}\right) \kappa_{\mathrm{i}} \frac{I_{m}^{\prime}\left(\kappa_{\mathrm{i}} a\right)}{I_{m}\left(\kappa_{\mathrm{i}} a\right)} \\
-\left[(\omega-\boldsymbol{k} \cdot \boldsymbol{U})^{2}-k_{z}^{2} v_{\mathrm{Ai}}^{2}\right] \kappa_{\mathrm{e}} \frac{K_{m}^{\prime}\left(\kappa_{\mathrm{e}} a\right)}{K_{m}\left(\kappa_{\mathrm{e}} a\right)}=0 .
\end{aligned}
$$

As we have already seen, the wave frequency $\omega$ is Dopplershifted inside the jet. We recall that depending on the values of the wave attenuation coefficients $\kappa_{\mathrm{i}}$ and $\kappa_{\mathrm{e}}$ in both media one can distinguish three major types of waves, namely pure surface waves when both coefficients are real, pseudosurface waves (or body waves as termed by Edwin \& Roberts 1983) for which $\kappa_{\mathrm{i}}$ is imaginary and $\kappa_{\mathrm{e}}$ real, and leaky waves in the opposite case when the energy is carried away from the flux tube (Cally 1986). In our case for the specified sound and Alfvén speeds, both modes turn out to be generally pseudosurface (body) waves that can, however, at some flow speeds become pure surface waves. The two tube speeds, respectively, are $c_{\mathrm{Ti}}=9.9 \mathrm{~km} \mathrm{~s}^{-1}$ and $c_{\mathrm{Te}}=231 \mathrm{~km} \mathrm{~s}^{-1}$. For the kink waves, one defines the kink speed (Edwin \& Roberts 1983)

$c_{\mathrm{k}}=\left(\frac{\rho_{\mathrm{i}} v_{\mathrm{Ai}}^{2}+\rho_{\mathrm{e}} v_{\mathrm{Ae}}^{2}}{\rho_{\mathrm{i}}+\rho_{\mathrm{e}}}\right)^{1 / 2}=\left(\frac{v_{\mathrm{Ai}}^{2}+\left(\rho_{\mathrm{e}} / \rho_{\mathrm{i}}\right) v_{\mathrm{Ae}}^{2}}{1+\rho_{\mathrm{e}} / \rho_{\mathrm{i}}}\right)^{1 / 2}$,

which is independent of sound speeds and characterises the propagation of transverse perturbations. Its value in our case is $74 \mathrm{~km} \mathrm{~s}^{-1}$.

\section{Wave dispersion diagrams}

It is obvious that dispersion Eq. (9) of either mode can be solved only numerically. Before starting that job, we normalise all velocities to the Alfvén speed $v_{\mathrm{Ai}}$ inside the jet thus defining the dimensionless phase velocity $V_{\mathrm{ph}}=v_{\mathrm{ph}} / v_{\mathrm{Ai}}$ and the Alfvén-Mach number $M_{\mathrm{A}}=U / v_{\mathrm{Ai}}$. The wavelength is normalised to the tube radius $a$ which means that the dimensionless wave number is $K=k_{z} a$. The calculation of wave attenuation coefficients requires the introduction of three numbers, notably the two ratios $\bar{\beta}=c_{\mathrm{s}}^{2} / v_{\mathrm{A}}^{2}$ correspondingly in the jet and its environment, and the ratio of the background magnetic field outside to that inside the flow, $b=B_{\mathrm{e}} / B_{\mathrm{i}}$, in addition to the density contrast, $\eta$. We 
recall that the two $\bar{\beta}$ s are 1.2 times smaller than the corresponding plasma betas in both media - the latter are given by the expressions $\beta_{\mathrm{i}, \mathrm{e}}=2 \bar{\beta}_{\mathrm{i}, \mathrm{e}} / \gamma$. Thus, with listed in Sect. 2 the specific speeds in both media and $\eta=0.01$, the input parameters in the numerical procedure are

$\eta=0.01, \quad \bar{\beta}_{\mathrm{i}} \cong 0.02, \quad \bar{\beta}_{\mathrm{e}} \cong 5.86, \quad b \cong 0.36, \quad$ and $\quad M_{\mathrm{A}}$.

The value of the Alfvén-Mach number, $M_{\mathrm{A}}$, naturally depends on the value of the streaming velocity $U$. Our choice of this value is $100 \mathrm{~km} \mathrm{~s}^{-1}$, which yields $M_{\mathrm{A}}=1.43$. With these input values, we calculate the dispersion curves of first kink waves and then sausage ones.

\subsection{Kink waves}

We start by calculating the dispersion curves of kink waves assuming that the angular wave frequency $\omega$ is real. As a reference, we first assume that the plasma in the flux tube is static, i.e., $M_{\mathrm{A}}=0$. The dispersion curves, which present the dependence of the normalised wave phase velocity on the normalised wave number, are in this case shown in Fig. 1. One can recognise three types of waves: a sub-Alfvénic slow magnetoacoustic wave (in red colour) labelled $\mathrm{C}_{\mathrm{Ti}}$ (which is actually the normalised value of $c_{\mathrm{Ti}}$ to $v_{\mathrm{Ai}}$ ), an almost Alfvén wave labelled $c_{k}$ (the green curve), and a family of super-Alfvénic waves (the magenta dispersion curves). We note that one can obtain by numerically solving Eq. (9) the mirror images (with respect to the zeroth line) of the $c_{k}$-labelled dispersion curve, as well as of the fast super-Alfvénic waves - both being backward propagating modes that are not plotted in Fig. 1. The next Fig. 2 shows how all these dispersion curves change when the plasma inside the tube flows. One sees that the flow first shifts upwards the almost Alfvén wave now labelled $c_{k}^{h}$, as well as high-harmonic super-Alfvénic waves - where both of the altered types of waves are depicted with points style lines in Fig. 2. Second, the slow magnetoacoustic wave (with label $\mathrm{C}_{T i}$ in Fig. 2) is replaced by two, now, super-Alfvénic waves, whose dispersion curves (in cyan and orange colours) are collectively labelled $\mathrm{C}_{\mathrm{Ti}}$. These two waves have practically constant normalised phase velocities equal to 1.289 and 1.571 , respectively, which are the $\left(M_{\mathrm{A}} \mp c_{\mathrm{Ti}}^{0}\right)$-values, where $c_{\mathrm{Ti}}^{0}$ is the normalised magnitude of the slow magnetoacoustic wave at $M_{\mathrm{A}}=0$. Unsurprisingly, one gets a $c_{k}$-labelled curve which is the mirror image of the $\mathrm{c}_{\mathrm{k}}^{\mathrm{h}}$-labelled curve. That is why this curve is plotted in green and, as can be seen, it is now a forward propagating wave that has, however, a smaller normalised phase velocity than that of its sister $\mathrm{c}_{\mathrm{k}}^{\mathrm{h}}$-labelled points-style-line dispersion curve. Moreover, there appears to be a family of generally backward propagating waves (below the $c_{k}^{l}$-labelled curve) plotted in blue colour that can similarly be considered as a mirror image of the highharmonic super-Alfvénic waves. The most interesting waves especially for the type II spicules seems to be the waves labelled $c_{k}$. It would be interesting to see whether these modes can become unstable at some, say critical, value of the Alfvén-Mach number $M_{\mathrm{A}}$. To study this, we have to assume that the wave frequency is complex, i.e., $\omega \rightarrow \omega+\mathrm{i} \bar{\gamma}$, where $\bar{\gamma}$ is the expected instability growth rate. Thus, the dispersion equation becomes complex (complex wave phase velocity and real wave number) and the solving a transcendental complex equation is generally a difficult task (see Acton 1990).

Before starting to derive a numerical solution of the complex version of Eq. (9), we can simplify that equation. Bearing in mind that the plasma beta inside the jet is very small $\left(\beta_{\mathrm{i}} \cong 0.024\right)$

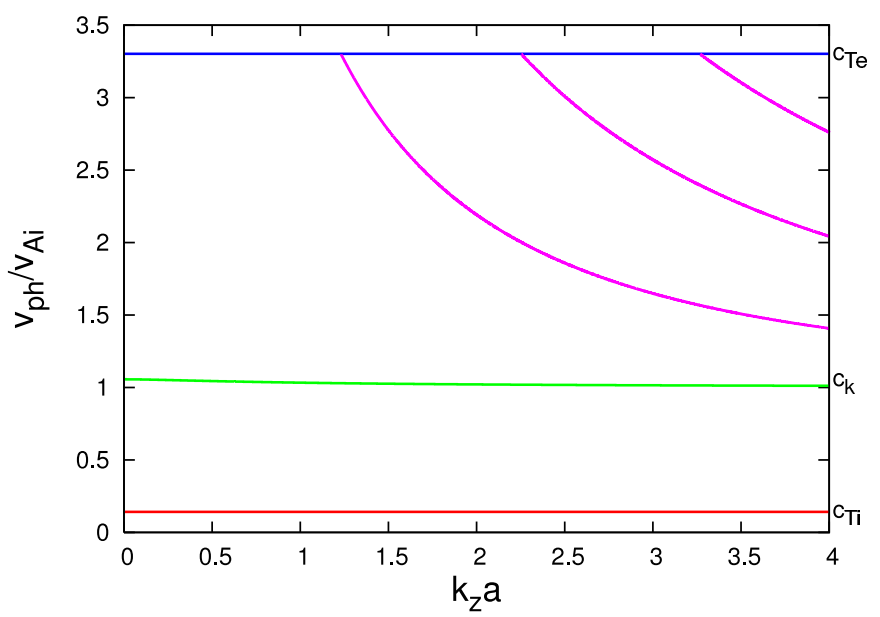

Fig. 1. Dispersion curves of kink waves propagating along the flux tube at $M_{\mathrm{A}}=0$.

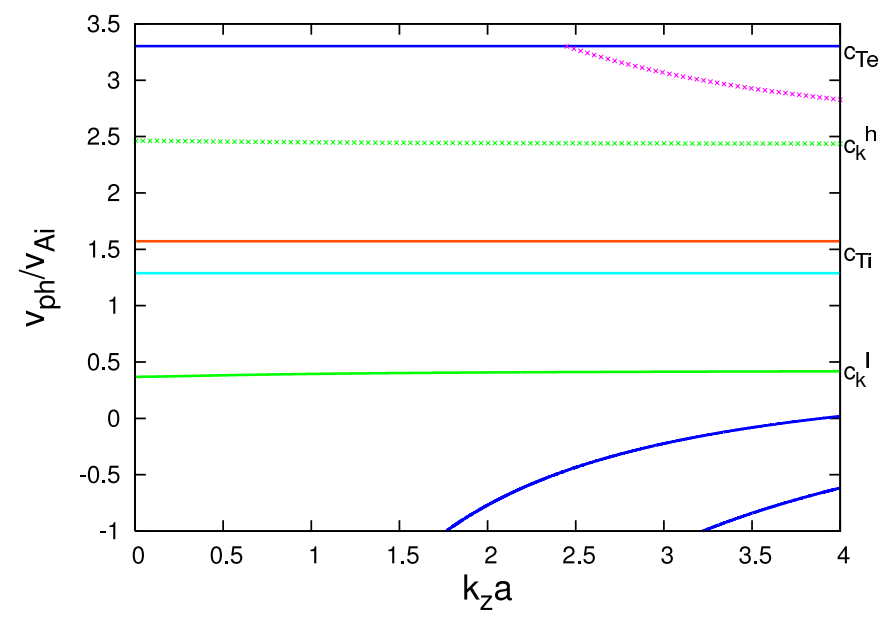

Fig. 2. Dispersion curves of kink waves propagating along the flux tube at $M_{\mathrm{A}}=1.43$.

and that of the surrounding medium quite high (of order 7), we can treat the jet as a cool plasma and the environment as a hot incompressible fluid. We point out that according to the numerical simulation of spicules by Matsumoto \& Shibata (2010) the plasma beta at heights greater than $2 \mathrm{Mm}$ is of the order of 0.03-0.04 (consider Fig. 4 of their paper). For cool plasma $c_{\mathrm{s}} \rightarrow 0$, hence the normalised wave attenuation coefficient $\kappa_{\mathrm{i}} a=\left[1-\left(V_{\mathrm{ph}}-M_{\mathrm{A}}\right)^{2}\right]^{1 / 2} K$, while in the incompressible environment $c_{\mathrm{s}} \rightarrow \infty$ and the corresponding attenuation coefficient is simply equal to $k_{z}$, i.e., $\kappa_{\mathrm{e}} a=K$. Under these circumstances the simplified dispersion equation of kink waves takes the form

$$
\begin{aligned}
\left(V_{\mathrm{ph}}^{2} \eta-b^{2}\right)[1 & \left.-\left(V_{\mathrm{ph}}-M_{\mathrm{A}}\right)^{2}\right]^{1 / 2} \frac{I_{1}^{\prime}\left(\kappa_{\mathrm{i}} a\right)}{I_{1}\left(\kappa_{\mathrm{i}} a\right)} \\
- & {\left[\left(V_{\mathrm{ph}}-M_{\mathrm{A}}\right)^{2}-1\right] \frac{K_{1}^{\prime}(K)}{K_{1}(K)}=0, }
\end{aligned}
$$

where we recall that $\kappa_{\mathrm{i}} a=\left[1-\left(V_{\mathrm{ph}}-M_{\mathrm{A}}\right)^{2}\right]^{1 / 2} K$, and the normalised wave phase velocity $V_{\mathrm{ph}}$ is a complex number. We note that this simplified version of the dispersion equation of the kink waves closely reproduces the dispersion curves labelled $c_{k}$ in Figs. 1 and 2.

To investigate the stability/instability status of the kink waves, we numerically solve Eq. (10) using the Müller method 
I. Zhelyazkov: Magnetohydrodynamic waves and their stability status in solar spicules

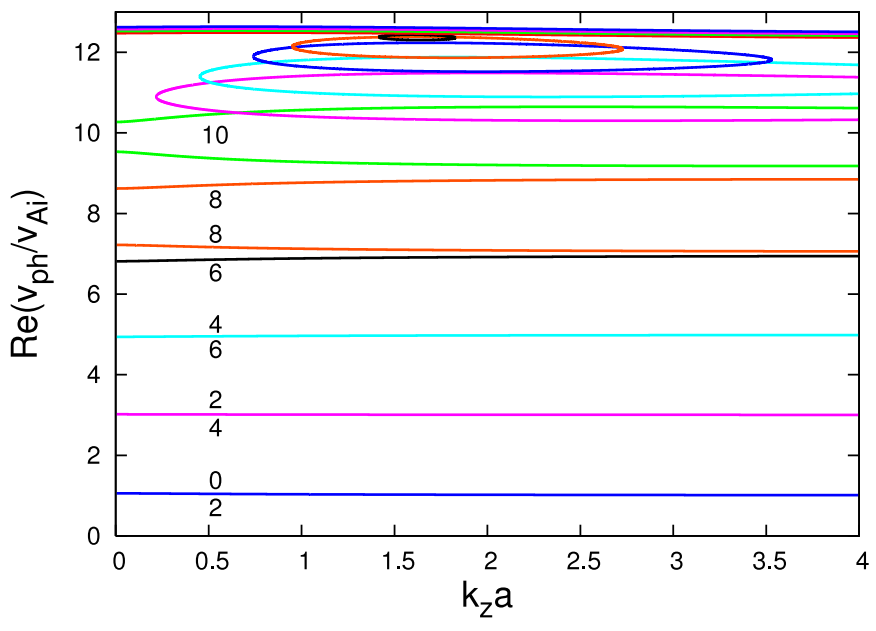

Fig. 3. Dispersion curves of kink waves propagating along the flux tube at various values of $M_{\mathrm{A}}$.

(Muller 1956) to find the complex roots at fixed input parameters $\eta=0.01$ and $b=0.36$, and varying Alfvén-Mach numbers $M_{\mathrm{A}}$ from zero to some reasonable numbers. Before starting any numerical procedure for solving the aforementioned dispersion equation, we note that for each input value of $M_{\mathrm{A}}$ one can get two $c_{\mathrm{k}}$-dispersion curves one of which (for relatively small magnitudes of $M_{\mathrm{A}}$ ) has a normalised phase velocity roughly equal to $M_{\mathrm{A}}-1$ and a second dispersion curve associated with dimensionless phase velocity equal to $M_{\mathrm{A}}+1$. These curves are similar to the dispersion curves labelled $c_{k}^{l}$ and $c_{k}^{h}$ in Fig. 2. The numerical solutions of Eq. (10) are shown in Fig. 3. For $M_{\mathrm{A}}=0$, except for the dispersion curve with normalised phase velocity approximately equal to 1 , one can find a dispersion curve with normalised phase velocity close to -1 - that curve is not plotted in Fig. 3. Similarly, for $M_{\mathrm{A}}=2$ one obtains a curve at $V_{\mathrm{ph}}=1$ and another at $V_{\mathrm{ph}}=3$, and so on. With increasing the magnitude of the Alfvén-Mach number, the kink waves change their structure - for small numbers being pseudosurface (body) waves and for $M_{\mathrm{A}}>4$ becoming pure surface modes. Another effect associated with the increase in $M_{\mathrm{A}}$, is that, for instance at $M_{\mathrm{A}} \geqslant 8$, the shapes of pairs of dispersion curves begin to visibly change as can be seen in Figs. 3 and 4. The most interesting observation is that for $M_{\mathrm{A}} \geqslant 11$ both curves start to merge and at $M_{\mathrm{A}}=12$ they form a closed dispersion curve. The ever increasing of $M_{\mathrm{A}}$ yields yet smaller closed dispersion curves - the smallest one depicted in Fig. 4 corresponds to $M_{\mathrm{A}}=12.5$. All these dispersion curves present stable propagation of the kink waves. However, for $M_{\mathrm{A}} \geqslant 12.6$ we obtain a new family of wave dispersion curves that correspond to an unstable wave propagation. We plot in Fig. 4 four curves of that kind that have been calculated for $M_{\mathrm{A}}=12.6,12.65,12.7$, and 12.75 , respectively. The growth rates of the unstable waves are shown in Fig. 5. The instability that arises is of the Kelvin-Helmholtz type. We recall that the Kelvin-Helmholtz instability, which is named after Lord Kelvin and Hermann von Helmholtz, can occur when velocity shear is present within a continuous fluid, or when there is a sufficient velocity difference across the interface between two fluids (Chandrasekhar 1961). In our case, we have the second option and the relative jet velocity $U$ plays the role of the necessary velocity difference across the interface between the spicule and its environment.

The main outstanding question is whether one can really observe such an instability in spicules. The answer to that question is obviously negative - to register the onset of Kelvin-Helmholtz

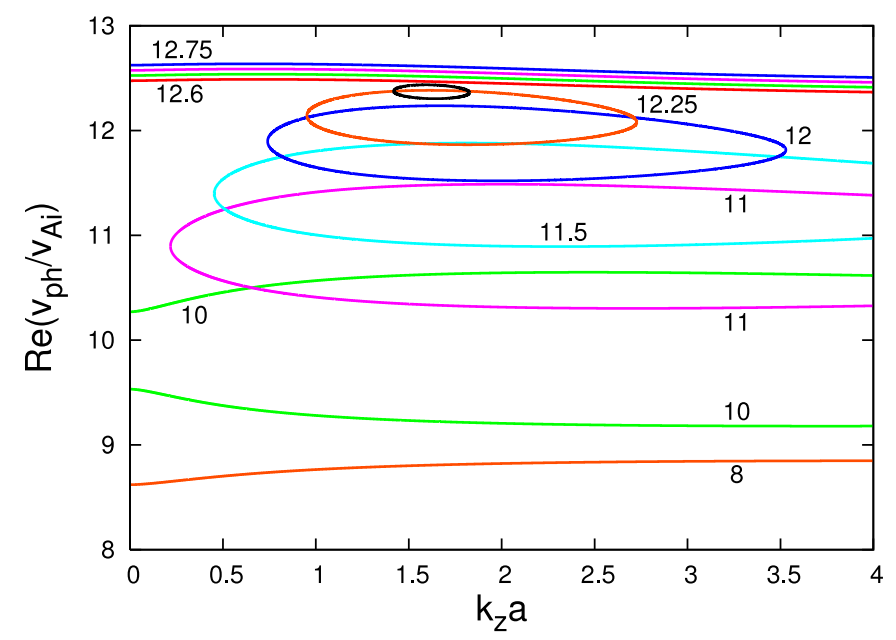

Fig. 4. Dispersion curves of kink waves propagating along the flux tube for relatively large values of $M_{\mathrm{A}}$.

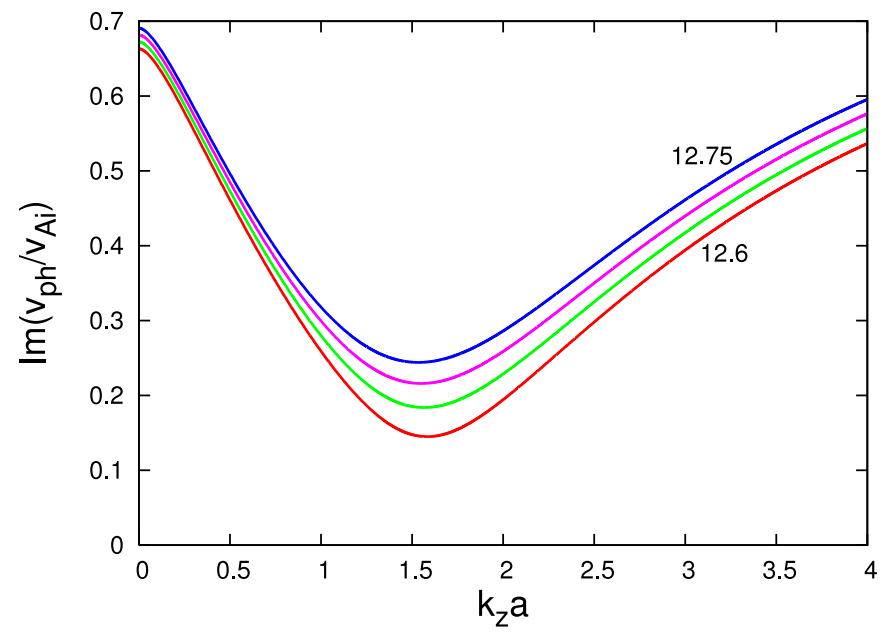

Fig. 5. Growth rates of unstable kink waves propagating along the flux tube at values of $M_{\mathrm{A}}$ equal to $12.6,12.65,12.7$, and 12.75 , respectively.

instability of kink waves travelling on a type II spicule, one would need to observe jet velocities of the order of or higher than $882 \mathrm{~km} \mathrm{~s}^{-1}$ ! If we assume that the density contrast, $\eta$, possesses a value of 0.02 and the ratio of the background magnetic fields, $b$, is equal to 0.35 (which may be deduced from a slightly different set of characteristic sound and Alfvén speeds in both media), the critical Alfvén-Mach number at which the instability starts is a little bit lower (equal to 8.9) but the corresponding jet speed is still too high $\left(=712 \mathrm{~km} \mathrm{~s}^{-1}\right)$ to be registered in a spicule. The value of 712 was computed under the assumption that the Alfvén speed inside the jet is $80 \mathrm{~km} \mathrm{~s}^{-1}$. Very similar dispersion curves and growth rates of unstable kink waves were obtained for cylindrical jets when both media were treated as incompressible fluids. In that case, dispersion Eq. (10) becomes a quadratic equation that provides solutions for the real and imaginary parts of the normalised wave phase velocity in closed forms (see Zhelyazkov 2010, 2011). It is astonishing that for our jet with $b=0.36$ and $\eta=0.01$ the quadratic dispersion equation yields a critical Alfvén-Mach number for the onset of a Kelvin-Helmholtz instability equal to 12.5 , which is lower than its magnitude obtained from Eq. (10). With this new critical Alfvén-Mach number, the required jet speed for the instability onset is $875 \mathrm{~km} \mathrm{~s}^{-1}$. It is worth mentioning that for the same 


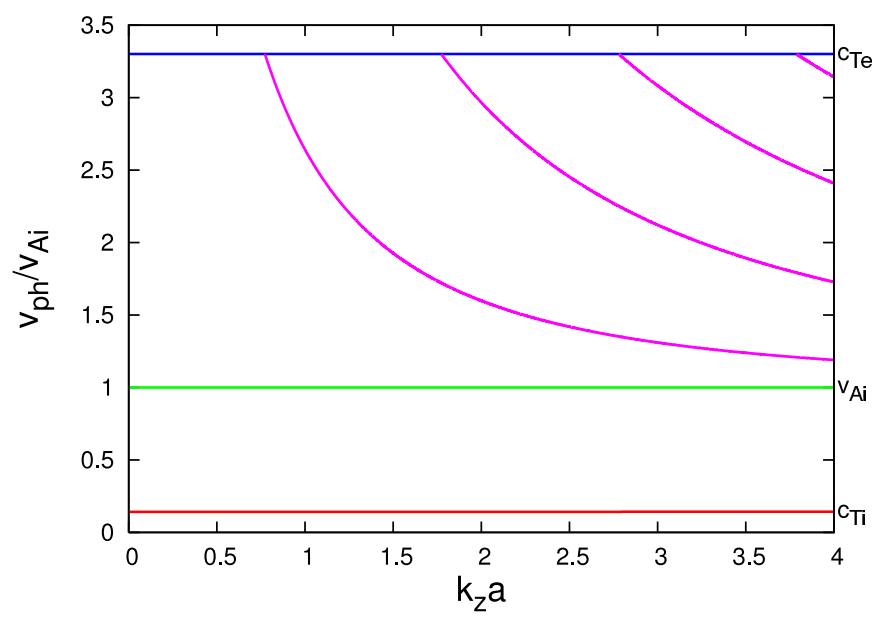

Fig. 6. Dispersion curves of sausage waves propagating along the flux tube at $M_{\mathrm{A}}=0$.

$\eta=0.01$, but for $b=1$ (equal background magnetic fields), the quadratic equation yields a much higher critical Alfvén-Mach number $(=15.65)$, which means that the critical jet speed grows up to $1096 \mathrm{~km} \mathrm{~s}^{-1}$. This consideration shows that both the density contrast, $\eta$, and the ratio of the constant magnetic fields, $b$, are equally important in determining the critical Alfvén-Mach number. Moreover, since Eq. (10) and its simplified form of a quadratic equation yield almost similar results (both for dispersion curves and growth rates when kink waves become unstable) firmly corroborates the correctness of the numerical solutions to the complex dispersion Eq. (10).

\subsection{Sausage waves}

The dispersion curves of sausage waves both in a static and in a flowing plasma shown in Figs. 6 and 7 are very similar to those of kink waves (compare with Figs. 1 and 2). The latter curves were calculated from dispersion Eq. (9) with azimuthal mode number $m=0$ for the same input parameters as in the case of kink waves. The main difference is that the $c_{k}$-labelled green dispersion curve is replaced by a curve corresponding to the Alfvén wave inside the jet. We note that the dispersion curve in Fig. 7 corresponding to a normalised phase velocity of the order of 0.5 (more exactly of 0.43 ) is labelled $v_{A i}^{\prime}$ because it can be considered as the one dispersion curve of the $(1.43 \pm 1)$-curves that can be derived from the dispersion equation. As in the case of kink waves, the dispersion curve corresponding to the higher speed has the label $v_{\mathrm{Ai}}^{\mathrm{h}}$. Here we also get two almost dispersionless curves collectively labelled $\mathrm{C}_{\mathrm{Ti}}$ (in the same colours, cyan and orange, as in Fig. 2) with normalised wave phase velocities equal to 1.289 and 1.571 . One of those curve starting at 1.2886 decreases to 1.2875 , while the other slightly increases starting at 1.5714 and finishing at 1.5725 . The same holds for the two similar curves in Fig. 2.

When examining the stability properties of sausage waves as a function of the Alfvén-Mach number $M_{\mathrm{A}}$, we use the same Eq. (10) while changing the order of the modified Bessel functions from 1 to 0 . As in the case of kink waves we are interested primarily in the behaviour of the waves whose phase velocities are multiples of the Alfvén speed. The results of numerical calculations of the complex dispersion equation are shown in Fig. 8. It turns out that for all reasonable Alfvén-Mach numbers the waves are stable. This is unsurprising because the same conclusion was drawn by solving precisely the complex

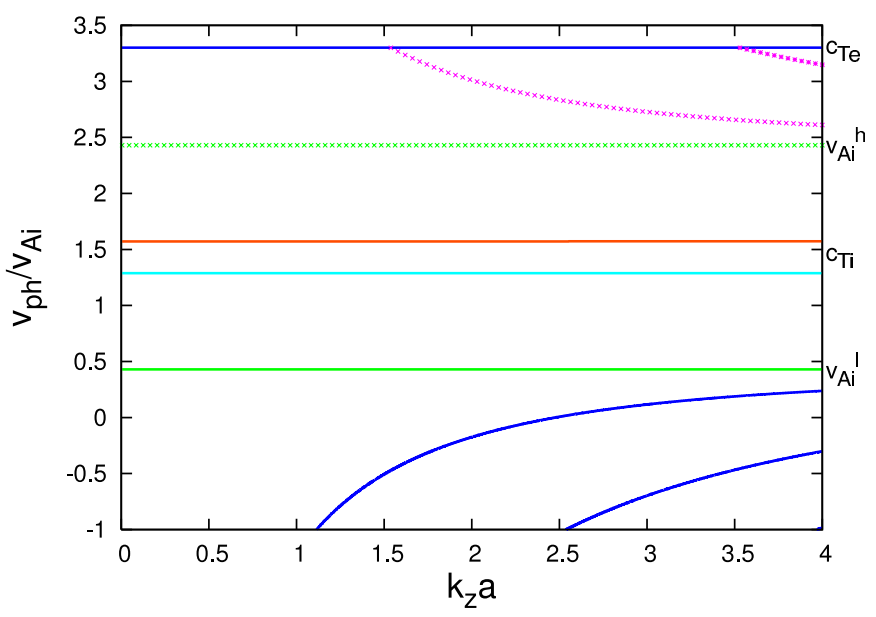

Fig. 7. Dispersion curves of sausage waves propagating along the flux tube at $M_{\mathrm{A}}=1.43$.

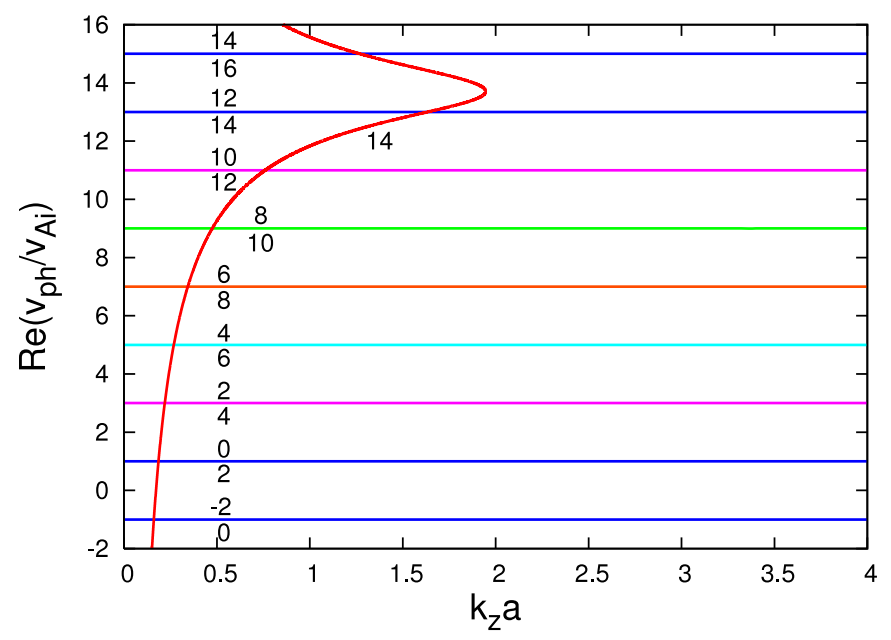

Fig. 8. Dispersion curves of sausage waves propagating along the flux tube at various values of $M_{\mathrm{A}}$.

dispersion equation governing the propagation of sausage waves in incompressible flowing cylindrical plasmas (see Zhelyazkov 2010, 2011). In Fig. 8, almost all dispersion curves have two labels: one for the $\left(\mathrm{M}_{\mathrm{A}}-1\right)$-labelled curve at given $M_{\mathrm{A}}$ (the label is below the curve), and second for the $\left(\mathrm{M}_{\mathrm{A}}^{\prime}+1\right)$-labelled curve associated with the corresponding $\left(M_{\mathrm{A}}^{\prime}=M_{\mathrm{A}}-2\right)$-value (the label is above the curve). This labelling is quite complex because for all $M_{\mathrm{A}}$ we find dispersion curves that overlap: for instance, the higher-speed dispersion curve (i.e., that associated with the $\left(M_{\mathrm{A}}+1\right)$-value) for $M_{\mathrm{A}}=0$ coincides with the lower-speed dispersion curve (i.e., that associated with the $\left(M_{\mathrm{A}}-1\right)$-value) for $M_{\mathrm{A}}=2$. In contrast to the kink waves that for $M_{\mathrm{A}}>4$ are pure surface modes, the sausage waves can be both pseudosurface (body) and pure surface modes, or one of the pair can be a surface mode while the other is a pseudosurface one. For example, all dispersion curves for $M_{\mathrm{A}}=0$ and 8 correspond to the pseudosurface (body) waves, while the curves' pair associated with $M_{\mathrm{A}}=4$ describes the dispersion properties of pure surface waves. For the other Alfvén-Mach numbers, one of the waves is a pseudosurface and the other is a pure surface. However, there is a "rule": if, for instance, the higher-speed wave with $M_{\mathrm{A}}=10$ is a pseudosurface mode, the lower-speed wave for $M_{\mathrm{A}}=12$ is a pure surface wave. We finish the discussion of sausage waves with the following conclusion: with 
increasing Alfvén-Mach number $M_{\mathrm{A}}$, the initially independent high-harmonic waves and their mirroring counterparts begin to merge - this is clearly seen in Fig. 8 for $M_{\mathrm{A}}=14-$ the resulting dispersion curve is in red colour. A similar dispersion curve can be obtained for $M_{\mathrm{A}}=12$, too; the merging point of the corresponding two high-harmonic dispersion curves lies, however, at around $k_{z} a=4.1$, i.e., beyond the limits of the plot. It is also evident that in the long wavelength limit the bottom part of the red-coloured dispersion curve describes a backward propagating sausage pseudosurface (body) wave. Another peculiarity of the same dispersion curve is the circumstance that for the range of dimensionless wave numbers between 1 and 2, one can have two different wave phase velocities. Which one is detected, the theory cannot predict.

\section{Conclusion}

We now summarise the main findings of our paper. We have studied the dispersion properties and the stability of the MHD normal modes running along the length of type II spicules. The latter have been modelled as straight cylindrical jets of ideal cool plasma surrounded by a warm/hot fully ionized medium. The wave propagation has been investigated in the context of standard magnetohydrodynamics by using linearised equations for the perturbations of the basic quantities: mass density, pressure, fluid velocity, and wave magnetic field. The derived dispersion equations describe the well-known kink and sausage mode influenced by the presence of spicules' moving plasma. The streaming plasma is characterized by its velocity $\boldsymbol{U}$, which is directed along the background magnetic fields $\boldsymbol{B}_{\mathrm{i}}$ and $\boldsymbol{B}_{\mathrm{e}}$ inside the jet and in its environment, respectively. An alternative and more convenient way of specifying the jet is by defining the Alfvén-Mach number: the ratio of jet speed to the Alfvén speed inside the jet, $M_{\mathrm{A}}=U / v_{\mathrm{Ai}}$. The key parameters controlling the dispersion properties of the waves are the so-called density contrast, $\eta=\rho_{\mathrm{e}} / \rho_{\mathrm{i}}$, the ratio of the two background magnetic fields, $b=B_{\mathrm{e}} / B_{\mathrm{i}}$, and the two ratios of the squared sound and Alfvén speeds, $\bar{\beta}_{\mathrm{e}}=c_{\mathrm{se}}^{2} / v_{\mathrm{Ae}}^{2}$ and $\bar{\beta}_{\mathrm{i}}=c_{\mathrm{si}}^{2} / v_{\mathrm{Ai}}^{2}$. How does the jet change the dispersion curves of both modes (kink and sausage waves) in a static-plasma flux tube? The answers to that question are as follows.

The flow shifts upwards the specific dispersion curves, the kink-speed curve for kink waves, and the Alfvén-speed curve for sausage waves, as well as high-harmonic fast waves of both modes. The sub-Alfvénic tube speed inside the jet, $c_{\mathrm{Ti}}$, belongs to two waves with normalised phase velocities equal to $M_{\mathrm{A}} \mp c_{\mathrm{Ti}} / v_{\mathrm{Ai}}$. One also observes such a duplication of the $c_{\mathrm{k}}{ }^{-}$ or $v_{\mathrm{Ai}}$-speed curve of kink or sausage waves. Below the lowerspeed $c_{\mathrm{k}}-$ or $v_{\mathrm{Ai}}$-curve, there is a set of dispersion curves that are a mirror image of the high-harmonic fast waves. We note that the flow does not affect the $c_{\mathrm{Te}}$-speed dispersion curve associated with the tube velocity in the environment.

For a typical set of characteristic sound and Alfvén speeds in both media (the jet and its environment) at relatively small Alfvén-Mach numbers, both modes are pseudosurface (body) waves. With increasing $M_{\mathrm{A}}$, some of them become pure surface waves. For kink waves, this finding is valid for $M_{\mathrm{A}}>4$.

The kink waves running along the jet can become unstable when the Alfvén-Mach number $M_{\mathrm{A}}$ exceeds some critical value. That critical value depends upon the two input parameters, $\eta$ and $b$; the increase in the density contrast decreases the magnitude of the critical Alfvén-Mach number, whilst the increase in the background magnetic fields ratio leads to an increase in the critical $M_{\mathrm{A}}$. For our choice of parameters $(\eta=0.01$ and $b=0.36$ ), the value of the critical $M_{\mathrm{A}}$ is 12.6 . This means that the speed of the jet must be at least $882 \mathrm{~km} \mathrm{~s}^{-1}$ to ensure the onset of the Kelvin-Helmholtz instability of propagating kink waves. Such high speeds of type II spicules have not yet been detected. If we take a twice greater value of $\eta(=0.02)$ and almost the same magnitude of $b(=0.35)$, the critical $M_{\mathrm{A}}$ is smaller (equal to 8.9) but still too high to permit unstable wave propagation - the required flow speed is $712 \mathrm{~km} \mathrm{~s}^{-1}$. A rough criterion for the appearance of the Kelvin-Helmholtz instability of kink waves is the satisfaction of an inequality suggested by Andries $\&$ Goossens (2001), which in our notation reads

$M_{\mathrm{A}}>1+b / \sqrt{\eta}$

This criterion provides more reliable predictions for the critical $M_{\mathrm{A}}$ when $b \approx 1$ (see Zhelyazkov 2010 ).

The onset of the Kelvin-Helmholtz instability for kink surface waves running along a cylindrical jet is preceded by a substantial reorganization of wave dispersion curves. As we increase the Alfvén-Mach number, the pairs of high- and low-speed curves (look at Fig. 4) begin to merge transforming into closed dispersion curves. After a further increase in $M_{\mathrm{A}}$, these closed dispersion curves become smaller - this is an indication that we have reached the critical $M_{\mathrm{A}}$ at which the kink waves are subjected to the Kelvin-Helmholtz instability - the unstable waves propagate across the entire $k_{z} a$-range having growth rates that depend upon the value of the current $M_{\mathrm{A}}$. We note that this behaviour has been observed for kink waves travelling on flowing solar-wind plasma (Zhelyazkov 2010, 2011).

We have found that the sausage waves are unaffected by the Kelvin-Helmholtz instability. This conclusion was also previously drawn for sausage modes in a flowing solar-wind plasma (Zhelyazkov 2010, 2011).

As we have seen, very high jet speeds are required to ensure that the Kelvin-Helmholtz instability occurs for kink waves propagating in type II spicules associated with a subsequent triggering of Alfvén wave turbulence, hence the possibility that this mechanism is responsible for chromospheric/coronal heating has to be excluded. However, a twist in the magnetic field of the flux tube or its environment may have the effect of lowering the instability threshold (see Bennett et al. 1999; Zaqarashvili et al. 2010) and eventually lead to the triggering of the Kelvin-Helmholtz instability. According to Antolin \& Shibata (2010), a promising way to ensure spicules'/coronal heating is by means of the mode conversion and parametric decay of Alfvén waves generated by magnetic reconnection or driven by the magnetoconvection at the photosphere. However, spicules can be considered as Alfvén wave resonant cavities (Hollweg 1981; Leroy 1981) and as Matsumoto \& Shibata (2010) claim, the waves of the period around 100-500 s can transport a large amount of wave energy to the corona. In all cases, the question of whether large coronal spicules can reach coronal temperatures remains open - for a discussion from an observational point of view we refer to Madjarska et al. (2011).

Acknowledgements. The author is indebted to Temury Zaqarashvili for fruitful discussions concerning waves' propagation in spicules and critical reading of the manuscript. Our sincere thanks also go to the referee for his detailed review, constructive criticism, and useful suggestions.

\section{References}

Acton, F. S. 1990, Numerical Methods That (Usually) Work, Mathematical Association of America, Washington, D.C., Chap. 14

Andries, J., \& Goossens, M. 2001, A\&A, 368, 1083 
A\&A 537, A124 (2012)

Antolin, P., \& Shibata, K. 2010, ApJ, 712, 494

Athay, R. G. 2000, Sol. Phys., 197, 31

Athay, R. G., \& Holzer, T. E. 1982, ApJ, 255, 743

Beckers, J. M. 1968, Sol. Phys., 3, 367

Beckers, J. M. 1972, ARA\&A, 10, 73

Bennett, K., Roberts, B., \& Narain, U. 1999, Sol. Phys., 185, 41

Cally, P. S. 1986, Sol. Phys., 103, 277

Centeno, R., Bueno, J. T., \& Ramos, A. A. 2010, ApJ, 708, 1579

Chandrasekhar, S. 1961, Hydrodynamic and Hydromagnetic Stability (Oxford: Clarendon Press), Chap. 11

de Pontieu, B., McIntosh, S., Hansteen, V. H., et al. 2007, PASJ, 59, S655

De Pontieu, B., McIntosh, S. W., Carlsson, M., et al. 2011, Science, 331, 55

Edwin, P. M., \& Roberts, B. 1983, Sol. Phys., 88, 179

He, J.-S., Tu, C.-Y., Marsch, E., et al. 2009, A\&A, 497, 525

Hollweg, J. V. 1981, Sol. Phys., 70, 25

Kukhianidze, V., Zaqarashvili, T. V., \& Khutsishvili, E. 2006, A\&A, 449, L35

Kudoh, T., \& Shibata, K. 1999, ApJ, 514, 493

Leroy, B. 1981, A\&A, 97, 245

Madjarska, M. S., Vanninathan, K., \& Doyle, J. D. 2011, A\&A, 532, L1

Matsumoto, T., \& Shibata, K. 2010, ApJ, 710, 1857
Moore, R. L., Sterling, A. C., Cirtain, J. W., \& Falconer, D. A. 2011, ApJ, 731 L18

Muller, D. A. 1956, Math. Tables Other Aids Comput., 10, 208

Nakariakov, V. M. 2007, Adv. Space Res., 39, 1804

Sterling, A. C. 2000, Sol. Phys., 196, 79

Sterling, A. C., Moore, R. L., \& DeForest, C. E. 2010, ApJ, 714, L1

Tavabi, E., Koutchmy, S., \& Ajabshirizadeh, A. 2011, New Astron., 16, 296

Terra-Homen, M., Erdélyi, R., \& Ballai, I. 2003, Sol. Phys., 217, 199

van Ballegooijen, A. A., Asgari-Targhi, M., Cranmer, S. R., \& DeLuca, A. A. 2011, ApJ, 736, 3

Zaqarashvili, T. V. 2011, in 3rd School and Workshop on Space Plasma Physics, ed. I. Zhelyazkov, \& T. Mishonov, AIP Conf. Proc., 1356, 106

Zaqarashvili, T. V., \& Erdĺyi, R. 2009, Space Sci. Rev., 149, 355

Zaqarashvili, T. V., Khutsishvili, E., Kukhianidze, V., \& Ramishvili, G. 2007, A\&A, 474, 627

Zaqarashvili, T. V., Días, A. J., Oliver, R., \& Ballester, J. L. 2010, A\&A, 516, A84

Zhelyazkov, I. 2010, Plasma Phys. Control. Fusion, 52, 065008

Zhelyazkov, I. 2011, in 3rd School and Workshop on Space Plasma Physics, ed. I. Zhelyazkov, \& T. Mishonov, AIP Conf. Proc., 1356, 138 